\title{
Knowledge, Attitude and Practice of Type 2 Diabetic Patients with Chronic Kidney Disease: A literature review
}

\author{
Mohammed Alvis Zibran ${ }^{1 *}$, Masoud Mohammadnezhad ${ }^{2}$, Rachel Page ${ }^{3}$, Sabiha Khan ${ }^{4}$ \\ ${ }^{1}$ Master in Public Health, School of Public Health and Primary Care, Fiji National University, Fiji Islands \\ ${ }^{2}$ Associate Professor, School of Public Health and Primary Care, Fiji National University, Fiji Islands \\ ${ }^{3}$ Associate Professor, School of Health Sciences, Massey University, New Zealand \\ ${ }^{4}$ Lecturer, School of Public Health and Primary Care, Fiji National University, Fiji Islands
}

*Corresponding Author: Mohammed Alvis Zibran, School of Public Health and Primary Care, Fiji

National University, Fiji Islands. Email: alviszibran@gmail.com

\begin{abstract}
Abs tract
Introduction: Having adequate knowledge, attitude and practice (KAP) about Type 2 Diabetes Mellitus (T2DM) and Chronic Kidney Disease (CKD) can lead to a reduction in this burden of disease.

Aim: The purpose of this review is to provide the level of KAP in T2DM patients with CKD and identify determinants of KAP in this particular population.

Method: Various medical databases including; Hinari, Scopus, MEDLINE, PubMed, CINAHL and Google Scholar were searched systematically to identify the articles pertaining to KAP in T2DM patients with CKD. There were no limitations on study type, time or place for this review though inclusion and exclusion criteria were applied.

Results: 11 of the 12 articles found poor knowledge among T2DM patients with CKD while only 2 of the 3 articles showed good attitude and 3 of the 5 articles showed poor practice. From the 9 studies which looked at determinants of KAP, apart from young, employed people with higher education having better knowledge scores, there were nil other major links identified.
\end{abstract}

Conclusion: T2DM patients with CKD have poorknowledge but unclear attitude and practice. Determinants of KAP in this particular population are not well researched and are a potential for further studies.

Keywor ds: Knowledge, Attitude, Practice, Type 2 Diabetes Mellitus, Chronic Kidney Disease

\section{INTRODUCTION}

Type 2 Diabetes Mellitus (T2DM) is a metabolic syndrome whereby the body is unable to use insulin, resulting in state of high blood sugars which has multiple eventual complications - of particular interest for this review being Chronic Kidney Disease (CKD) which is majorly $(44 \%)$ caused by the former. $^{(1,2)}$ CKD is defined as estimated Glomerular Filtration Rate (eGFR) of less than $60 \mathrm{~mL} / \mathrm{min} / 1.73 \mathrm{~m}^{2}$ for 3 months or more. ${ }^{(3)}$ Globally, 1 in 7 adults suffer from CKD and it is the $9^{\text {th }}$ leading cause of death worldwide which is a humongous economic burden on the health system of any nation for that matter. ${ }^{(2)}$

T2DM needs to be addressed due to the horrific consequences of this disease. T2DM doubles a person's risk of cardiovascular event such as heart attack or stroke, damages eyes (retinopathy) plus nerves (neuropathy) and is the leading cause of CKD. ${ }^{(4,5)}$ In terms of mortality, diabetes is $9^{\text {th }}$ leading cause of death globally. ${ }^{(6)}$

Knowledge about a disease is the understanding, grasp, belief or familiarity regarding the illness and it is a factor that influences the adherence to preventive or therapeutic treatment. ${ }^{(7)}$ It can have a substantial influence on patient's version of disease together with its course and treatment. ${ }^{(8)}$ This shows that proper knowledge of a disease is vital and is at the crux of entire patient management process.

Patient's attitude about a disease represents their stance, viewpoint, perspective, opinion or approach which is reflected in their behavior as either positive or negative in terms of disease management process. ${ }^{(9)}$ Patient's attitude towards their disease affects not only their immediate disease status but also the chronic 
outcomes which show that the level of attitude is directly linked with the illness plus probability of its complications. ${ }^{(10)}$ On the contrary, positive attitude helps to improve the physical health while negative attitude obviously deters health-seeking behaviors. ${ }^{(11)}$

Practice in diabetic patients with CKD implies the actual conversion of knowledge and attitude regarding the disease into action for prevention or management as advised by their health-care provider. The level of practice determines the extent of control of patient's clinical conditions and is an important indicator for overall patient management, compliance and frequency of complications. ${ }^{(12,13)}$

If the diabetics with CKD were supposedly well-versed with the causative factors, there is a high chance that their knowledge about the disease determinants would positively influence their attitude towards the disease process. ${ }^{(14)}$ Consequently, this shall be reflected in their practices towards adopting healthier alternatives that shall lead to delay in disease progression to the final stage of complications which are expensive to the patient, his/her family and the health sector as a whole.

\section{Methodology}

The following Databases were used to search for articles regarding the topic of study - Hinari, Scopus, EBSCO HOST Super Package [MEDLINE], PubMed, CINAHL and Google Scholar. The search criteria used for this purpose - Keywords (in titles and/or abstracts) were used with search strategies - ("Chronic kidney disease" OR "chronic renal disease" OR "chronic renal impairment" OR "chronic renal insufficiency") AND "Knowledge, Attitude and Practice", ("Type 2 Diabetes" OR "adult diabetes" OR "Non-Insulin Dependent Diabetes"). There was not any year or location limitation in searching the studies. Those studies which did not address all aspects of KAP in only T2DM patients with CKD were excluded. Additionally, this review did not take into account the articles that have CKD attributable to other causes apart from T2DM.

Once the articles were retrieved from the various databases, they were grouped under the major sub-headings to compile the literature review. Firstly, all articles which showed the link between T2DM and its progression to CKD were considered as the first part of the review. Then, the studies which focused on level of
KAP in T2DM patients with CKD were considered as a second part of the review so as to enable the identification of the individual aspects of level of KAP. Finally, all articles pertaining to determinants of KAP in this particular population were grouped under the final heading to identify the common links between them and compare and contrast the differences or similarities.

In particular, caution was taken to avoid misrepresenting the information provided in the main articles. All articles used for the review were individually added to the reference list to avoid being missed out.

\section{LITERATURE REVIEW}

\subsection{T2DM and CKD}

T2DM is one the 4 major NCDs (others being Cardiovascular Disease, Chronic Respiratory Disease and Cancer) that are contributing to the NCD crisis globally, regionally and nationally. ${ }^{(1)}$ The prevalence of T2DM in adults (aged more than 18 years) worldwide almost doubled from 1980 to 2016 - from $4.7 \%$ to $8.5 \%$, especially in middle and low-income nations. ${ }^{(6)}$ In the Western Pacific Region (WPR), the prevalence of DM2 in 2013 was $8.6 \%$ while half of those with this disease remain undiagnosed. ${ }^{(15)} \mathrm{A}$ report on NCD in WPR stated that almost all Pacific Island Countries had a prevalence of T2DM of at least $10 \% .^{(16)}$

Knowledge or awareness of T2DM can shape a person's attitude which shall be reflected in their practice towards appropriate preventive measures in order to avoid the T2DM-related complications and premature mortality. ${ }^{(14)}$ Consequently, it is vital to explore the KAP in T2DM patients with CKD in order to determine the high-risk groups so as to enable the healthsystem to deliver a strategic approach for awareness, prevention and targeted programs to maximize the efficiency of the health-care delivery process.

T2DM is the major cause of CKD accounting for $44 \%$ of all cases of CKD. ${ }^{(2,6)}$ The deadly combination of T2DM and CKD needs to be addressed especially in countries which are resource-strained in terms of health-services. A study in France by Detournay, et al. ${ }^{(17)}$ showed that $40 \%$ of the patients in the last stage of CKD had T2DM and poor control of T2DM raised risks of kidney disease and progression. The authors also found that addressing T2DM intensively via medications to achieve good 
sugar control will not only reduce chances of kidney damage but also decrease cardiovascular-related disease/death. This shows that the progression of T2DM needs to be halted as early as possible to ensure these patients escape the aforementioned risks.

Another study done in USA by Nelson, et al. ${ }^{(18)}$ showed that $33 \%$ of the general population with End-Stage Kidney Disease (ESKD) had T2DM while Pima Indians of USA had an even higher incidence of both T2DM and ESKD but the determinants and disease progression were similar to the rest of the population. The authors explained the pathophysiology of renal damage in T2DM by stating that hyperfiltration of kidneys later leads to eventual rapid decline in kidney function and hence irreversible kidney damage.

Once the kidneys reach a certain stage whereby they can no longer accommodate for the increased loads of filtration, their compensation mechanism fails. The period of time elapsed from time of diagnosis of T2DM and onset of kidney failure is difficult to determine as there are numerous factors which act synergistically to compound the failure mechanism. Unfortunately, by the time patients become symptomatic with kidney failure, it is already too late as the renals have sustained irreversible damage and it is almost impossible to salvage them. There are numerous signs and symptoms that manifest in people with T2DM and CKD (as it progresses to stage of complications) and recognition and treatment of these can influence the disease progress.

Li Vecchi, et al. ${ }^{(19)}$ studied the prevalence of one of the complications of CKD - anemia, in T2DM patients and non diabetic patients and they found that the prevalence of anemia was significantly higher $(\mathrm{p}<0.05)$ in T2DM patients with CKD compared to non-diabetic patients with CKD. This is just one example to show the lethal link between T2DM and CKD which the authors had re-iterated in their findings. In another study by Packham, et al. ${ }^{(20)}$, T2DM patients with proteinuria were shown to have high risks of cardiovascular deaths but this was lower than their overall 3-year risk of ESKD. This study shows that the link between T2DM and kidney failure are more common than the link between T2DM and other cardiac conditions like heart attack and stroke but this does not undermine the significance of the latter association between T2DM and cardiovascular system.

The association between T2DM and CKD has been explored so far and it has been established that T2DM is strongly associated with CKD and ESKD. However, moving onto the other aspects of this deadly combination, there are cardiovascular manifestaitions which are worth mentioning. A study done in T2DM Chinese patients by Hsieh, et al. ${ }^{(21)}$ showed that T2DM and CKD were associated with higher risk of Coronary Artery Disease. This cardiovascular link has been mentioned earlier on as well in the study by Packham, et al. ${ }^{(20)}$ Similarly, the worsening of CKD in T2DM lead to a significantly $(p<0.001)$ higher risk of a major cardiovascular event in another study by Ragot, et al. ${ }^{(22)}$

\subsection{Le vel of KAP towards the risk of CKD in T2DM Patients}

There have been numerous studies done to evaluate patient's level of KAP towards their diseases but only few studies were found that looked specifically at KAP in T2DM patients with CKD. Patients can have good knowledge and attitude about their disease but this does not necessarily mean that they will have good practice as well. Similarly, they can have poor knowledge but good attitude and practice and so forth.

The essence of mentioning this is that every patient is unique and the overall KAP of a representative sample of a particular population can provide useful information on the level of KAP in the sample. The researcher needs to be careful before generalizing this finding to the rest of that population but the bottom line remains that a fair idea is achieved (if the study design is properly chosen) about overall level of KAP in the study sample which can provide useful information on which of the 3 components of KAP is/are low and consequently need to be addressed as a healthcare intervention program.

Poor knowledge but good attitude and practice was found in a cross-sectional study conducted in Malaysia by Yusoff, Yusof \& $\mathrm{Kueh}^{(23)}$ using self-administered questionnaires to identify KAP in patients with medical conditions towards the risks for CKD and also to determine its link with 7 determinants (discussed in detail in next section) - sex, age, race, education level, 
marital status, occupation and monthly family income.

The study selected 103 patients by convenient sampling (admitted to the 4 medical and surgical wards of a single tertiary care hospital) and found that approximately $69.9 \%$ patients had poor knowledge but $68.9 \%$ had good attitude while $88.3 \%$ patients had good practice towards risk for CKD. Hence, poor knowledge in majority of the participants was the main concern raised by the authors in this study.

Similarly, poor awareness and knowledge was found in a qualitative study in United Sates of America (USA) by Kazley, et al (24) which aimed to look at the African-American patients' level of knowledge of their CKD in 2008 at a single medical renal center whereby $20 \mathrm{CKD}$ patients were selected by non-random sampling and assigned to 4 focus groups to answer 5 open-ended questions regarding CKD. After analyzing the data, the authors found that majority of the patients (percentage not specified) had poor knowledge and awareness regarding CKD risk factors, prevention and management. Consequently, the recommendation was to focus on targeting this particular ethnic group (high-risk) for awareness and prevention programs in order to avoid rise in new CKD cases.

On the same note, poor knowledge was found in a prospective study in USA by Whaley-Connell, et al ${ }^{(25)}$ which had aimed to measure level of awareness of CKD and its link to the final stage of CKD amongst 109,285 patients from 20002009. The results showed that $26 \%$ of the participants had CKD but only $9 \%$ of them were aware of CKD. The interesting part was that the patients who were aware of their disease had significantly higher risk for progressing to final stage of CKD and death. As a result, the authors stated that mere awareness does not necessarily improve disease progression which is a contrast to the other findings as aforementioned.

Yet another descriptive study in USA conducted by Johnson, Zimmerman, Welch, Hertzog, Pozehl \& Plumb ${ }^{(26)}$ with a convenient sample of 85 people with DM, HTN and CKD, collected data using telephone or face-to-face interviews, found that the average knowledge of CKD to be $62.8 \%$. The authors claimed that this percentage was lower than those found in earlier studies in CKD patients.
Poor level of knowledge seems to be evident in most of the studies though the level of attitude and practice fluctuates. This could be a worthwhile observation since the universality of the findings of low level of knowledge could possibly point to the fact that probably the overall/global health system is failing in its part to adequately educate its T2DM patients with CKD.

Moving on, poor knowledge and practice but good attitude was seen in a cross-sectional study in Northern Tanzania by Stanifer, et al ${ }^{(27)}$ using KAP Survey questionnaire developed by Stanifer, et al ${ }^{(28)}$. This study had aimed to firstly assess KAP amongst people with either CKD or at risk of CKD and also to identify the determinants of KAP (which are discussed in the next section). The authors found that average level of knowledge was low (average score of 3.28 out of $10 ; 95 \%$ CI 2.94, 3.63) but the attitude was high $-97 \%$. However, the problem identified by the authors was that almost $80 \%$ participants had opted for poor practices which included traditional healers/medications or even self-management.

As aforementioned, poor knowledge was once again the main concern raised by the authors and this was compounded by another important result - poor level of practice in participants of the study. A good level of attitude is not able to do much good if the overall practice is low but knowing that attitude is good can help the health professionals to build upon that particular aspect and eventually influence the practice of T2DM patients with CKD.

Similar findings of poor knowledge and awareness was found in a study by $\mathrm{Li}$, et al ${ }^{(29)}$ who aimed to look at level of awareness of CKD in the general population in all the 50 states of USA using a telephone survey in 2011. Since $2.5 \%$ of the general public was aware of CKD from the sample size of 404,718 people, the authors concluded that the awareness was very low and hence there was a need to promote this in the general public. However, this survey was not done in patients who were T2DM with CKD but rather in the general public and hence the extremely low level of knowledge was seen. The rationale for choosing this study was to show that level of knowledge about T2DM and $\mathrm{CKD}$ is relatively lower in general population compared to T2DM patients with CKD, though both groups manifest poor overall knowledge. 
Another cross-sectional study in USA by Tuot, et al ${ }^{(30)}$ looked at knowledge and awareness of CKD in 1852 adults with CKD and reported a low level of CKD awareness - only $16 \%$ at most knew about CKD. There was no attitude or practice measured in this study but the poor level of knowledge was consistent with all the other literature.

On the contrary, high/good knowledge and attitude but poor practice was seen in another study. The CKD Screening Index developed by Khalil, et al (31) was used by Khalil \& Abdalrahim ${ }^{(12)}$ to identify KAP of 740 patients (without CKD but having either T2DM or Hypertension (HTN), family history of CKD and age more than 18 years) towards prevention and detection of CKD in Jordan. This crosssectional study, conducted from 2012-2013 using cluster sampling to recruit patients from the 4 main cities, found that though the overall knowledge was high (80\%), 50\% were unaware of CKD manifestations. Approximately, 78.7\% patients had good attitude while $65 \%$ had good practices hence the authors showed concern on improving knowledge so as to be able to impact the level of practice.

Furthermore, low/poor knowledge and attitude was evident in a cross-sectional study in Iran by Roomizadeh, et al. ${ }^{(32)}$ which aimed to determine the knowledge of CKD plus T2DM and HTN in general population using self-administered questionnaires to 748 participants who were selected by non-random (convenience) sampling. The authors found that general knowledge of CKD was very low $(12 \%)$ while an alarming $60 \%$ subjects had poor attitude to CKD. However, this study did not make an attempt to measure level of attitude and practice in the participants. Nonetheless, poor knowledge is once again the main issue detected in this study like most other studies mentioned in the review.

Similarly, poor knowledge and attitude was found in a community-based survey in Indiana (USA) involving 1465 people from 4 ethnic minority communities (African-American, Hispanic, American Indian and Asian/Pacific Islander) which aimed to find the level of KAP and beliefs regarding CKD via the use of selfadministered questionnaires. ${ }^{(33)}$ The authors found that almost $40 \%$ participants had poor attitude while a similar proportion had good knowledge.
Interestingly, this article looked at ethnic minorities whereby the Pacific Islanders were involved. However, those Pacific Islanders involved in the study were residents of USA and hence it is difficult to say whether the finding can be generalized to residents from Pacific Island Countries (PIC) in the Western Pacific Region.

Low level of knowledge was once again seen in a nested cross-sectional study in Australia which aimed to find out the knowledge of renal disease in 852 T2DM adults who were part of a longitudinal study (AusDiab). ${ }^{(34)}$ The authors reported that there was a very low level of knowledge (average of $20 \%$ ) of CKD and hence recommended to educate patients so as to allow early detection of risk factors for CKD.

If knowledge is universally low, education of the patients becomes a crucial element for consideration which has been recommended in most of the studies. However, mere increase in knowledge does not necessarily equate to good attitude and practice as these are three separate components that need to be addressed accordingly.

A nested cross-sectional American study by Finkelstein, et al. ${ }^{(35)}$ also found poor knowledge in the study subjects CKD patients (half of whom had T2DM). In this particular study involving $676 \mathrm{CKD}$ patients, the authors aimed to determine level of "perceived knowledge" regarding last stage of CKD using selfadministered questionnaires. The findings showed that $35 \%$ had no knowledge regarding CKD treatment options while almost $66 \%$ had poor knowledge.

Unfortunately, there were no studies done in PIC to identify level of KAP in T2DM patients with CKD. Knowing the level of KAP in this population is very vital as it enables us to identify the key areas for health intervention. For instance, if a study had found that T2DM patients with CKD in a particular country had poor level of knowledge, the health sector could utilize this vital information to introduce various health strategies to raise knowledge. Similarly, if attitude or practice were found to be low/poor, appropriate steps can be taken to address them accordingly in order to reduce the burden of complication of T2DM and CKD. On the contrary, if any component of KAP was found to be high, re-enforcing the health program in that particular element while strengthening the other components via re-distribution of 
resources can enable rise in overall level of KAP and ultimately reduce the expensive complications of T2DM and CKD.

\subsection{Determinants of KAP in T2DM patients with CKD}

There may be numerous factors that affect KAP in T2DM patients with CKD but only few studies were identified that actually tested the link between them. The level of KAP can be affected by lot of variables and it is crucial to quantify the association between these determinants and KAP as this will give an idea about which factors are significant and consequently can be targeted for future interventions in order to raise KAP.

To begin with, Yusoff, Yusof \& Kueh (23) identified the major significant associations for poor knowledge were females, age more than 60 years, non-Tertiary education, unemployment and low family income $(\mathrm{p}<0.05)$ while significant level of good attitude was seen in females, age more than 30 years, married and employed patients $(\mathrm{p}<0.05)$ and in terms of good practice, significant links were seen only in the married patients $(\mathrm{p}=0.008)$.

This study shows that, generally, older age and poor socio-economic status (SES) had lower level of knowledge. This is quite logical since lower SES is usually linked with poor health outcomes. SES takes into account 3 major factors - education level, income and employment status and hence it is common to see that low levels of these 3 variables lowers the chances of seeking, accessing and utilizing health-services. Consequently, this group of people is usually unaware of disease processes, treatment options and so forth which lead to low/poor level of knowledge.

Moving on, in USA, in terms of ethnicity, African-Americans were at high risk for CKD and had poor knowledge but this was not quantified. ${ }^{(24)}$ This notion was supported by another study which stated that AfricanAmericans had significantly high association ( $p$ $<$ 0.001) with poor knowledge. ${ }^{(35)}$ Unfortunately, the ethnicities from PIC were not tested to identify its link with KAP and this makes it difficult to generalize the findings of this and other similar western studies (in terms of ethnicity at least) to the Pacific setting which has a completely different ethnic composition.

In Tanzania, high knowledge score was seen in age less than 60 years, urban-dwellers, higher education level (strongest association with knowledge) and unemployed people. ${ }^{(27)}$ However, neither attitude nor knowledge was significantly linked with demographical characteristics in this study, though males were significantly linked to only one aspect of the practice component. The surprising aspect to note is that unemployed people had higher knowledge which is a contrast to the findings by Yusoff, Yusof \& Kueh (23) who had stated otherwise - employed people having higher knowledge scores.

In another USA study, poor knowledge and awareness of CKD was significantly linked to unemployment, older age, males (less than 54 years) and females (more than 60 years). ${ }^{(29)}$ Once again poor SES was linked to low level of education but the conflict persists whether males or females dominated the knowledge aspect.

The Jordan study showed that older age, male sex and high monthly income significantly increased level of practice while male sex improved attitude scores and high level of education raised the knowledge score. ${ }^{(12)}$ The findings from this study do not refute the results from the other literature and basically builds upon the findings to support the link of some of the variables to KAP.

The Iranian study showed a non-significant link between good knowledge of CKD and female gender, age less than 65 and higher education. ${ }^{(32)}$ However, the authors recommended future programs to target the elderly and patients with poor SES since their findings were consistent with other studies. It is evident from the conclusion of this particular study that despite failing to get significant link between the variables, the final recommendation is presented which aligns with the findings from the other literature as discussed above, though some conflict remains on the actual link between gender and KAP.

Similarly, higher education, older age, being employed and Hispanic ethnicity significantly increased the knowledge, awareness and attitude in ethnic minorities in USA. ${ }^{(33)}$ This study shows high SES is linked with higher level of knowledge but the issue of ethnicity and KAP is still left to be clarified.

Additionally, an Australian study showed males $(\mathrm{p}=0.002)$, higher level of education $(\mathrm{p}<$ $0.001)$ and age below $60(\mathrm{p}<0.001)$ had better knowledge of CKD. ${ }^{(34)}$ Once again, higher SES 
is positively associated with better level of education though the link between gender and KAP is not concurrent with other literature.

The determinants influence the level of KAP and it is essential to identify these determinants since it will enable us to identify specific target groups for our public health interventions in order to increase and influence the overall value of KAP in future. Quite unfortunately, nil studies were found that addressed the link between the determinants and level of KAP in the Pacific. Knowing the determinants of KAP in T2DM with CKD patients is not just for academic purpose as there is a bigger picture we have to focus on - identifying the target groups towards whom our resources for public health programs will be aimed at so as to reduce the huge burden of disease attributed to T2DM and CKD. Once the link between the variables and KAP is established, focused public health programs (awareness/interventions/policies) can be targeted at the identified high-risk patients in an attempt to reduce the burden of the complications of CKD in DM 2.

\section{CONClusion}

T2DM is the major cause of CKD and hence having proper KAP about these 2 diseases can enable better control and reduced complications which will lead to a decline in the overall NCD burden. Unfortunately, in T2DM patients with $\mathrm{CKD}$, the level of knowledge has been found to be quite low since majority (92\%) of the articles showed poor knowledge in this particular group. The attitude and practice levels cannot be ascertained as very few articles were found that looked at these 2 components and even so, there were contrasting views mentioned on their level which makes it difficult to conclude about the true level whether it is actually good or poor.

Determinants of KAP revealed that the younger age group, employed people and those with higher education level had good knowledge though there is uncertainty on the attitude and practice in these people. The other determinants also do not show a clear link with level of KAP and hence needs to be researched in future studies. Identifying the level of KAP and its determinants is very crucial since it gives an indication of the population's baseline state and helps to detect crucial factors that can be targeted through public health intervention programs in order to raise the level of KAP so as to reduce the burden of disease attributable to T2DM and CKD in this context.

\section{REFERENCES}

[1] WHO. WHO News - Fact Sheets: Diabetes Mellitus. World Health Organization. [Online] 2018. [Cited: February 18, 2018.] http://www.who.int/mediacentre/factsheets/fs 13 8/en/.

[2] CDC. National Chronic Kidney Disease Fact Sheet. Centers for Disease Control and Prevention. [Online] 2017. [Cited: February 18, 2018.] https://www.cdc.gov/kidneydisease/ pdf/ kidney_factsheet.pdf.

[3] Arora, Pradeep. Chronic Disease. Medscape. [Online] May 1, 2017. [Cited: February 10, 2018.] https://emedicine.medscape.com/article /238798-overview.

[4] Sarwar, N, et al., Diabetes mellitus, fasting blood glucose concentration, and risk of vasculardisease: a collaborative meta-analysis of 102 prospective studies. Emerging Risk Factors Collaboration. 9733, June 26, 2010, Lancet, Vol. 375, pp. 2212-22.

[5] Saran, R, et al., US Renal Data System 2014 Annual Data Report: Epidemiology of Kidney Disease in the United States. 3, July 2015, American Journal of Kidney Diseases: The Official Journal of The National Kidney Foundation, Vol. 66, p. 545.

[6] Media Centre - Diabetes Fact Sheet. World Health Organization. [Online] November 2017. [Cited: March 3, 2018.] http://www.who.int/ mediacentre/factsheets/fs 312/en/.

[7] Heydari, Abbas, Ziaee, Elaheh Sadat and Gazrani, Akram Relationship between Awareness of Disease and Adherence to Therapeutic Regimen among Cardiac Patients. . 1, January 2015, International Journal of Community Based Nursing and Midwifery, Vol. 3, pp. 23-30.

[8] Szymona-Pałkowska, Katarzyna, et al.,Knowledge of the Disease, Perceived Social Support, and Cognitive Appraisals in Women with Urinary Incontinence. November 22, 2016, Hindawi - BioMed Research International, Vol. 2016, p. 7.

[9] Rahaman , Shafiur Kh, et al., Knowledge, Attitude and Practices (KAP) Regarding Chronic Complications of Diabetes among Patients with Type 2 Diabetes in Dhaka. 3, 2017, International Journal of Endocrinology \& Metabolism, Vol. 15. doi: 10.5812/ije m. 12555.

[10] Chen, Ailing, et al., Attitudes Toward Diabetes Affect Maintenance of Drug-Free Remission in Patients With Newly Diagnosed Type 2 Diabetes After Short-Term Continuous Subcutaneous Insulin Infusion Treatment. 3, March 2012, Diabetes Care, Vol. 35, pp. 474481. 
[11] Framingham, Jane. Positive Thinking Improves Physical Health. PsychCentral. [Online] 2016. [Cited: February 22, 2018.] https://psychcentral.com/lib/the-power-ofpositive-thinking/.

[12] Khalil, Amani and Abdalrahim, M. Knowledge, attitudes, and practices towards prevention and early detection of chronic kidney disease. June 2014, International Nursing Review, pp. 237245.

[13] Mohammadi, Shooka, et al., Knowledge, Attitude and Practices on Diabetes Among Type 2 Diabetic Patients in Iran: A CrossSectional Study. 4, May 29, 2015, Science Journal of Public Health, Vol. 3, pp. 520-524.

[14] Cummings, E, et al., Knowledge attitude and practices of persons diagnosed with type II diabetes mellitus with regards to nephropathy as a complication. 2, 2014, South American Journal of Public Health, Vol. 2, pp. 151-167.

[15] Chan, JC, et al., Diabetes in the Western Pacific Region--past, present and future. 2, February 2014, Diabetes Research and Clinical Practice, Vol. 103, pp. 244-55.

[16] Young-soo, Shin. Noncommunicable Diseases in The Western Pacific Region - A profile. World Health Organization - Western Pacific Region. [Online] 2011. [Cited: March 3, 2018.].

[17] Detournay, B, et al., Chronic kidney disease in type 2 diabetes patients in France: Prevalence, influence of glycaemic control and implications for the pharmacological management of diabetes. 2, April 2012, Diabetes and Metabolism, Vol. 38, pp. 102-112. DOI: https://doi.org/10.1016/j.diabet.2011.11.005.

[18] Nelson, Robert G, et al., Development and Progression of Renal Disease in Pima Indians with Non-Insulin-Dependent Diabetes Mellitus. November 28, 1996, The New England Journal Of Medicine, Vol. 335, pp. 1636-1642.

[19] Li Vecchi, M, et al., Prevalence and Severity of Anaemia in Patients with Type 2 Diabetic Nephropathy and Different Degrees of Chronic Renal Insufficiency. 2, 2007, Nephron Clinical Practice, Vol. 105.

[20] Packham, DK, et al., Relative incidence of ESRD versus cardiovascular mortality in proteinuric type 2 diabetes and nephropathy: results from the DIAMETRIC (Diabetes Mellitus Treatment for Renal Insufficiency Consortium) database. 1, January 2012, American Journal of Kidney Disease, Vol. 59, pp. 75-83.

[21] Hsieh, MC, et al., Chronic Kidney Disease as a Risk Factor for Coronary Artery Disease in Chinese with Type 2 Diabetes. 2, 2008, American Journal of Nephrology, Vol. 28, pp. 317-323.
[22] Ragot, Stéphanie, et al., Dynamic Changes in Renal Function Are Associated With Major Cardiovascular Events in Patients With Type 2 Diabetes. 7, July 2016, Diabetes Care, Vol. 39, pp. 1259-1266.

[23] Yusoff, Dariah Mohd, Yusof, Junaiti and Kueh, Yee Cheng. Knowledge, Attitude And Practices of The Risk For Chronic Kidney Disease Among Patients In A Tertiary Teaching Hospital. December 27, 2016, The Malaysian Journal of Nursing, Vol. 8, pp. 3-11.

[24] Kazley, Abby S, et al., African American patient knowledge of kidney disease: A qualitative study of those with advanced chronic kidney disease. 4, October 21, 2014, SAGE Journals, Vol. 11, pp. 245-255.

[25] Whaley-Connell, Adam, et al., Awareness of Kidney Disease and Relationship to End-Stage Renal Disease and Mortality: Elsevier INc., 2012, The American Journal of Medicine, Vol. 125, pp. 661-669.

[26] Johnson, Michelle L, et al., Patient Activation With Knowledge, Self-Management And Confidence In Chronic Kidney Disease. 2016, Journal of Renal Care.

[27] Stanifer, John W, et al., Knowledge, Attitudes, and Practices Associated with Chronic Kidney Disease in Northern Tanzania: A CommunityBased Study. 6, July 9, 2016, PLoS ONE, Vol. 11. doi: 10.1371/journal.pone.0156336

[28] Stanifer, John W, et al., Development and Validation of a Cross Cultural Knowledge, Attitudes and Practices Survey Instrument for Chronic Kidney Disease in a Swahili-Speaking Population. 3, March 26, 2015, PLoS ONE, Vol. 10.

[29] Li, C haoyang, et al., Awareness of Kidney Disease among US Adults: Findings from the 2011 Behavioral Risk Factor Surveillance System. April 12, 2014, American Journal of Nephrology, Vol. 39, pp. 306-313.

[30] Tuot, Delphine S, et al., Chronic Kidney Disease Awareness Among Individuals with Clinical Markers of Kidney Dysfunction. August 2011, Clnical Journal of American Society of Nephrology, Vol. 6, pp. 1838-1844.

[31] Khalil, A A, et al., Development and psychometric evaluation of the chronic kidney disease screening index. 8, 2014, Renal Failure., Vol. 36, pp. 1200-1207.

[32] Roomizadeh, Peyman, et al., Limited knowledge of chronic kidney disease and its main risk factors among Iranian community: an appeal for promoting national public health education programs. 4, 2014, International Journal of Health Policy and Management, Vol. 2, pp. 161-166.

[33] Ryder, Priscilla T, et al., Knowledge, Attitudes, Behaviors, and Beliefs about Chronic Kidney 
Knowledge, Attitude and Practice of Type 2 Diabetic Patients with Chronic Kidney Disease: A Literature Review

Disease in Indiana's Minority Communities: A Community-Based Survey. May 2013, Scholarship and Professional Work - COPHS, Vol. 89.

[34] White, Sarah L, et al., Limited Knowledge of kidney disease in a survey of AusDiab study participants. 4, February 18, 2008, Medical Journal of Australia, Vol. 188.
[35] Finkelstein, Fredric O, et al., Perceived knowledge among patients cared for by nephrologists about chronic kidney disease and end-stage renal disease therapies. July 30, 2008, Kidney Internationa;, Vol. 74, pp. 11781184.

Citation: Mohammed Alvis Zibran, Masoud Mohammadnezhad, Rachel Page, Sabiha Khan. Knowledge, Attitude and Practice of Type 2 Diabetic Patients with Chronic Kidney Disease: A literature review. ARC Journal of Public Health and Community Medicine.2018; 3(2):19-27. DOI: dx.doi.org/ 10.20431/24560596.0302004

Copyright: (c) 2018 Authors. This is an open-access article distributed under the terms of the Creative Commons Attribution License, which permits unrestricted use, distribution, and reproduction in any medium, provided the original author and source are credited. 